Oncofertility Medical Practice 

Clarisa Gracia • Teresa K. Woodruff

Editors

\section{Oncofertility Medical Practice}

Clinical Issues and Implementation 


\author{
Editors \\ Clarisa Gracia, M.D., M.S.C.E. \\ Department of Obstetrics and Gynecology \\ Perelman School of Medicine \\ University of Pennsylvania \\ Philadelphia, PA, USA
}

\author{
Teresa K. Woodruff, Ph.D. \\ Thomas J. Watkins Professor \\ of Obstetrics \& Gynecology \\ Feinberg School of Medicine \\ Northwestern University \\ Chicago, IL, USA
}

ISBN 978-1-4419-9424-0

ISBN 978-1-4419-9425-7 (eBook)

DOI 10.1007/978-1-4419-9425-7

Springer New York Heidelberg Dordrecht London

Library of Congress Control Number: 2012940873

(C) Springer Science+Business Media New York 2012

This work is subject to copyright. All rights are reserved by the Publisher, whether the whole or part of the material is concerned, specifically the rights of translation, reprinting, reuse of illustrations, recitation, broadcasting, reproduction on microfilms or in any other physical way, and transmission or information storage and retrieval, electronic adaptation, computer software, or by similar or dissimilar methodology now known or hereafter developed. Exempted from this legal reservation are brief excerpts in connection with reviews or scholarly analysis or material supplied specifically for the purpose of being entered and executed on a computer system, for exclusive use by the purchaser of the work. Duplication of this publication or parts thereof is permitted only under the provisions of the Copyright Law of the Publisher's location, in its current version, and permission for use must always be obtained from Springer. Permissions for use may be obtained through RightsLink at the Copyright Clearance Center. Violations are liable to prosecution under the respective Copyright Law.

The use of general descriptive names, registered names, trademarks, service marks, etc. in this publication does not imply, even in the absence of a specific statement, that such names are exempt from the relevant protective laws and regulations and therefore free for general use.

While the advice and information in this book are believed to be true and accurate at the date of publication, neither the authors nor the editors nor the publisher can accept any legal responsibility for any errors or omissions that may be made. The publisher makes no warranty, express or implied, with respect to the material contained herein.

Printed on acid-free paper

Springer is part of Springer Science+Business Media (www.springer.com) 


\section{Foreword}

Oncofertility has emerged as an important field in reproductive medicine. With important advances in cancer treatment now allowing many men and women to live full and productive lives, reproductive concerns emerge as being of great importance for overall quality of life and fulfillment. Also, in recent years, we have witnessed advances in our understanding and appreciation of gonadal and gamete biology, allowing assisted reproductive technologies to offer great promise for preserving the reproductive function of cancer survivors.

This book, edited by Clarisa Gracia and Teresa Woodruff, is a state-of-the-art treatise on all clinical aspects of oncofertility. It is the third in a series of books on this subject, the first two dwelling on basic science and sociological and humanity issues. This multiauthored and multidisciplinary text, by leaders in the field, accomplishes its goal of providing clinicians and researchers the ability to help cancer survivors achieve a long-term quality of life.

Oncofertility Medical Practice: Clinical Issues and Implementation is timely, practical, and comprehensive and is divided into several important parts. Part I sets the stage in describing the fertility risks of cancer patients. Next, Part II provides several options for both males and females. In Part III, the care of the patient is highlighted in terms of social and bioethical issues which affect cancer survivors, as well as dealing with pregnancy. The remainder of this book is unique in that it provides very practical advice. Part IV explains the "nuts and bolts" of clinical practice in oncofertility, including chapters on setting up a program, patient navigation, and preparing an educational and research training program. In Part V, clinical cases in oncofertility are presented. The Appendix provides valuable resources for the clinician and researcher adapted from the National Physicians Cooperative of the Oncofertility Consortium. This includes information pertinent for IRB and FDA applications, patient consent forms, and letter templates to aid patients with issues of reimbursement for treatment. 
This excellent text is an invaluable resource and a "must read" for clinicians and researchers interested in oncofertility. The editors and authors need to be congratulated for their fine work.

Roger A. Lobo, M.D.

Professor of Obstetrics and Gynecology

Columbia University, Immediate Past President

American Society for Reproductive Medicine 


\section{Preface}

More than 100,000 females younger than 45 years of age are diagnosed with cancer annually; of these, approximately 12,400 are children younger than 20 years of age $[1,2]$. Over the past four decades, advances in cancer therapies, particularly chemotherapeutics, have led to dramatic improvements in survival [3]. Given that more patients are surviving their cancer, care has now expanded to include improving long-term health and quality of life. One of the most important quality-of-life issues in reproductive-age cancer survivors is the ability to have biological children [4-6]. Unfortunately, cancer therapies - including chemotherapy, radiotherapy, and some surgical procedures - increase the risk of infertility and gonadal failure in both males and females.

Given the reproductive risks of cancer therapies, as well as the improved longterm survival made possible by these therapies, there has been growing interest in expanding future reproductive options for cancer patients. Indeed, both cancer survivors and the medical community have acknowledged the importance of patient counseling and pursuit of fertility preservation options prior to starting potentially gonadotoxic cancer treatment. In 2006, the American Society of Clinical Oncology published recommendations on fertility preservation, stating, "As part of education and informed consent before cancer therapy, oncologists should address the possibility of infertility with patients treated during their reproductive years and be prepared to discuss possible fertility preservation options or refer patients to reproductive specialists" [7]. Similarly, the American Society of Reproductive Medicine has encouraged clinicians to offer cancer patients available technologies for fertility preservation, stating, "If damage to reproductive organs from treatment is unavoidable, cryopreserving gametes, embryos, or gonadal tissue may help to preserve fertility" [8]. Despite these recommendations, fertility preservation services continue to be underutilized [9].

The field of oncofertility is focused on addressing this unmet need in a multidisciplinary manner [10]. Indeed, the practice of oncofertility necessarily bridges a variety of disciplines, including oncology, urology, reproductive endocrinology, psychology, and philosophy. This book is intended to provide a comprehensive overview of the clinical aspects of oncofertility and is appropriately authored by 
experts in a variety of disciplines involved in providing fertility preservation options to cancer patients. It is primarily targeted to clinicians and researchers who wish to gain up-to-date information about the risks of cancer therapies, learn about options to preserve future reproductive potential in patients with cancer, understand the ethical and psychosocial issues impacting the practice of oncofertility, and learn strategies for setting up an oncofertility program. Two other books have been published that describe advances in oncofertility basic science research [2] and the perspectives of the social sciences and the humanities on fertility preservation for cancer patients [6]. Taken together, these three volumes serve as comprehensive and essential reading on the various facets of the field of oncofertility.

This book is divided into five parts. Part I provides current information regarding the reproductive risks of cancer therapies in females and males. Part II reviews available fertility preserving technologies from experts in the field. Standard and experimental options are presented for both male and female patients. Part III highlights important psychosocial and ethical issues in the field, with a particular focus on communication between patients and health-care providers. In addition, risks of pregnancy during and after a cancer diagnosis are discussed. Part IV includes practical guidance on how to establish an oncofertility clinical program and how to train future generations of oncofertility specialists. Valuable resources from the National Physician's Cooperative of the Oncofertility Consortium are presented in the Appendix, including templates for obtaining IRB approval for investigational fertility preservation strategies, marketing brochures, and online resources for patients and providers. The final section, Part V, includes clinical cases that highlight strategies and challenges in the care of the oncofertility patient.

As more clinicians become aware of the field of oncofertility and begin to offer their patients evidence-based counseling regarding the risks of cancer therapies and options available for having future biological children, we can expect to see improvements in the long-term quality of life of cancer survivors. We hope that the information and resources provided in this book help us to accomplish that goal.

\section{References}

1. Jemal A, et al. Cancer statistics, 2008. CA Cancer J Clin. 2008;58:71-96.

2. Woodruff TK, Snyder KA, eds. Oncofertility: fertility preservation for cancer survivors. New York: Springer; 2007.

3. Wingo PA, et al. Cancer incidence and mortality, 1973-1995: a report card for the U.S. Cancer. 1998;82:1197-1207.

4. Loscalzo MJ, Clark KL. The psychosocial context of cancer-related infertility. Cancer Treat Res. 2007;138:180-190.

5. Canada AL, Schover LR. The psychosocial impact of interrupted childbearing in long-term female cancer survivors. Psychooncology. 2010. doi:10.1002/pon.1875.

6. Woodruff TK, et al., eds. Oncofertility: ethical, legal, social, and medical perspectives. New York: Springer; 2007. 
7. Lee SJ, et al., American Society of Clinical Oncology. American Society of Clinical Oncology recommendations on fertility preservation in cancer patients. J Clin Oncol. 2006;24: 2917-2931.

8. Ethics Committee of the American Society for Reproductive Medicine. Fertility preservation and reproduction in cancer patients. Fertil Steril. 2005;83:1622-1628.

9. Lee $\mathrm{S}$, et al. Determinants of access to fertility preservation in women with breast cancer. Fertil Steril. 2011;95:1932-1936.

10. Woodruff TK. The Oncofertility Consortium-addressing fertility in young people with cancer. Nat Rev Clin Oncol. 2010;7:466-475. 



\section{Contents}

\section{Part I Fertility Risks for Cancer Patients}

1 Gonadotoxicity of Cancer Therapies in Pediatric and Reproductive-Age Females

Jennifer Levine

2 Gonadotoxicity of Cancer Therapies in Pediatric and Reproductive-Age Males

Jill P. Ginsberg

\section{Part II Options for Preserving Fertility}

3 Fertility Preservation in Males Landon Trost and Robert Brannigan

4 Embryo and Oocyte Banking Lynn M. Westphal and Jamie A.M. Massie

5 Ovarian Tissue Cryopreservation and Transplantation. Laxmi A. Kondapalli

6 The Role of In Vitro Maturation in Fertility Preservation

Peter S. Uzelac, Greg L. Christensen, and Steven T. Nakajima

7 Mitigating the Risk: The Role of Ovarian Transposition and Medical Suppression

Jaime M. Knopman and Nicole Noyes

\section{Part III Care of the Oncofertility Patient}

8 The Birds and the Bees and the Bank: Talking with Families About Future Fertility Amidst a Cancer Diagnosis Gwendolyn P. Quinn, Caprice A. Knapp, and Devin Murphy 
9 Addressing the Three Most Frequently Asked Questions of a Bioethicist in an Oncofertility Setting.

Lisa Campo-Engelstein

10 Pregnancy in Cancer Patients and Survivors Eileen Wang

11 Communication Between Oncofertility

Providers and Patients.

Jennifer Mersereau

\section{Part IV Oncofertility in Clinical Practice}

12 Setting Up an Oncofertility Program.

H. Irene Su, Lindsay Ray, and R. Jeffrey Chang

13 Patient Navigation and Coordination of Care for the Oncofertility Patient: A Practical Guide

Kristin Smith, Brenda Efymow, and Clarisa Gracia

14 Preparing an Interdisciplinary Workforce in Oncofertility: A Suggested Educational and Research Training Program Christos Coutifaris

\section{Part V Clinical Cases in Oncofertility and Resources}

15 Clinical Cases in Oncofertility 203 Clarisa Gracia

Appendices - Oncofertility Resources for the Clinician 225 Kate E. Waimey

Appendix A Sample IRB Protocol: Ovarian Tissue Freezing for Fertility Preservation in Women Facing a Fertility Threatening Medical Diagnosis or Treatment Regimen

Appendix B Sample Consent Form: Ovarian Tissue Freezing for Fertility Preservation in Adult Women Facing a Fertility Threatening Medical Diagnosis or Treatment Regimen .

Appendix C Sample IRB Protocol: Oocyte Banking For Fertility

Preservation In Women Facing A Fertility Threatening Medical Diagnosis or Treatment Regimen

Appendix D Sample Consent Form: Oocyte Banking for Fertility Preservation in Women Facing a Fertility Threatening Medical Diagnosis or Treatment Regimen 
Appendix E Letter Template: Provider Letter of Medical

Necessity for Fertility Preservation Procedures

Appendix F Letter Template: Patient Letter of Appeal

for Fertility Preservation Procedures Template

Appendix G Updating or Establishing Your FDA Registration.

Index 



\section{Contributors}

Robert Brannigan, M.D. Associate Professor, Department of Urology, Northwestern University, Feinberg School of Medicine, Chicago, IL, USA

Lisa Campo-Engelstein, Ph.D. Assistant Professor, Alden March Bioethics Institute, Albany Medical College, Albany, NY, USA

R. Jeffrey Chang, M.D. Professor and Director, Division of Reproductive Endocrinology, University of California, La Jolla, CA, USA

Greg L. Christensen, Ph.D., H.C.L.D. IVF Laboratory Director, University Women's Healthcare, University of Louisville, Louisville, KY, USA

Christos Coutifaris, M.D., Ph.D. Division of Reproductive Endocrinology and Infertility, Hospital at the University of Pennsylvania, Philadelphia, PA, USA

Brenda Efymow, R.N., B.S.N. Oncofertility Nurse Coordinator, Penn Fertility Care, Perelman School of Medicine at the University of Pennsylvania, Philadelphia, PA, USA

Jill P. Ginsberg, M.D. Cancer Survivorship Program, Children's Hospital of Philadelphia, Perelman School of Medicine at the University of Pennsylvania, Philadelphia, PA, USA

Clarisa Gracia, M.D., M.S.C.E. Assistant Professor, Department of Obstetrics and Gynecology, Perelman School of Medicine at the University of Pennsylvania, Philadelphia, PA, USA

Caprice A. Knapp, Ph.D. Assistant Professor, Department of Health Outcomes and Policy, University of Florida, Gainesville, FL, USA

Jaime M. Knopman, M.D. Division of Reproductive Endocrinology, New York University School of Medicine, New York, NY, USA

Laxmi A. Kondapalli, M.D., M.S.C.E. Assistant Professor, Section of Reproductive Endocrinology and Infertility, University of Colorado Denver, Aurora, CO, USA 
Jennifer Levine, M.D., M.S.W. Assistant Professor of Clinical Pediatrics, Division of Oncology, Columbia University Medical Center, New York, NY, USA

Jamie A.M. Massie, M.D. Obstetrics and Gynecology, Stanford University School of Medicine, Stanford, CA, USA

Jennifer Mersereau, M.D., M.S.C.I. Assistant Professor of Clinical Pediatrics, Division of Reproductive Endocrinology and Infertility, University of North Carolina, Chapel Hill, NC, USA

Janet McClaren, M.D., M.S.C.E. Division of Reproductive Endocrinology and Infertility, University of Alabama, Birmingham, AL, USA

Devin Murphy, M.S.W. Research Associate, Jonathan Jacques Children's Cancer Center, Miller Children's Hospital/HARBOR-UCLA, Long Beach, CA, USA

Steven T. Nakajima, M.D. Research Associate, Division of Reproductive Endocrinology and Infertility, Department of Obstetrics, Gynecology and Women's Health, University of Louisville, Louisville, KY, USA

Nicole Noyes, M.D. Professor, Division of Reproductive Endocrinology, New York University School of Medicine, New York, NY, USA

Gwendolyn P. Quinn, Ph.D. Associate Professor and Member, Health Outcomes and Behavior Program, Moffitt Cancer Center, Department of Oncologic Sciences, College of Medicine, University of South Florida, Tampa, FL, USA

Lindsay Ray, B.S.N. Reproductive Partners-UCSD Regional Fertility Center, La Jolla, San Diego, CA, USA

Kristin Smith Department of Obstetrics and Gynecology, Northwestern Medical Faculty Foundation, Chicago, IL, USA

H. Irene Su, M.D., M.S.C.E. Division of Reproductive Endocrinology, University of California, La Jolla, CA, USA

Landon Trost, M.D. Mayo Clinic, Rochester, MN, USA

Peter S. Uzelac, M.D. Medical Director, Napa Valley Fertility Center, Napa, CA, USA

Kate E. Waimey, Ph.D. Program Director, Oncofertility Consortium, Feinberg School of Medicine, Northwestern University, Chicago, IL, USA

Eileen Wang, M.D. Perelman School of Medicine at the University of Pennsylvania, Philadelphia, PA, USA

Lynn M. Westphal, M.D. Associate Professor, Obstetrics and Gynecology, Stanford University School of Medicine, Stanford, CA, USA

Teresa K. Woodruff, Ph.D. Thomas J. Watkins Professor of Obstetrics \& Gynecology and Director of the Oncofertility Consortium, Feinberg School of Medicine, Northwestern University, Chicago, IL, USA 